\title{
Spared and Impaired Olfactory Abilities after Thalamic Lesions
}

\author{
Lee Sela, ${ }^{1}$ Yaron Sacher, ${ }^{2,3}$ Corinne Serfaty, ${ }^{2,3}$ Yaara Yeshurun, ${ }^{1}$ Nachum Soroker, ${ }^{2,3 *}$ and Noam Sobel ${ }^{1 *}$ \\ ${ }^{1}$ Department of Neurobiology, The Weizmann Institute of Science, Rehovot 76100, Israel, ${ }^{2}$ Loewenstein Rehabilitation Hospital, Ra'anana 43100, \\ Israel, and ${ }^{3}$ Sackler Faculty of Medicine, Tel Aviv University, Ramat Aviv 69978, Israel
}

Olfactory information reaches olfactory cortex without a thalamic relay. This neuroanatomical substrate has combined with functional findings to suggest that, in olfaction, the typical thalamic role in sensory processing has shifted to the olfactory bulb or olfactory cortex. With this in mind, we set out to ask whether the thalamus at all plays a significant functional role in human olfaction. We tested olfactory function in 17 patients with unilateral focal thalamic lesions and in age-matched healthy controls. We found that thalamic lesions did not significantly influence olfactory detection but significantly impaired olfactory identification, and only right lesions altered olfactory hedonics by reducing the pleasantness of pleasant odors. An auditory control revealed that this shift in pleasantness was olfactory specific. These olfactory impairments were evident in explicit measures of perception, as well as in patterns of sniffing. Whereas healthy subjects modulated their sniffs in accordance with odorant content, thalamic patients did not. We conclude that, although the thalamus is not in the path of olfactory information from periphery to cortex, it nevertheless plays a significant functional role in human olfaction.

\section{Introduction}

Anatomical connectivity suggests a thalamic role in olfaction that is different from its role in other sensory modalities. Sensory information (e.g., vision and audition) typically projects from the periphery, via the thalamus, to cortex. Olfactory information, however, projects from olfactory epithelium to olfactory bulb, and from there directly to primary olfactory (piriform) cortex, without a thalamic relay (Haberly and Price, 1977; Scott et al., 1980). From primary olfactory cortex, information projects to the orbitofrontal cortex by two routes: a direct route and an indirect route via the mediodorsal (MD) nucleus of the thalamus (Price and Slotnick, 1983; Price, 1985). In other words, the place of the thalamus in the stream of processing is different in olfaction than in other senses. Two studies have indicated that, in olfaction, the typical thalamic role in sensory processing has been adopted by either the olfactory bulb (Kay and Sherman, 2007) or the primary olfactory cortex (Murakami et al., 2005). With this in mind, one may ask whether there remains a pronounced thalamic role in olfaction, and, if so, what is its functional nature?

One way to address this is by characterizing the influence of thalamic lesions on olfactory function. Studies in rodents have indicated that thalamic lesions do not influence odor detection but do influence olfactory discrimination (Eichenbaum et al., 1980; Slotnick and Kaneko, 1981) and complex learning tasks

\footnotetext{
Received May 5, 2009; revised July 23, 2009; accepted Aug. 7, 2009.

This work was supported by a European Research Council FP7 Ideas grant (200850).

*Na.S. and No.S. contributed equally to this work.

Correspondence should be addressed to either Lee Sela or Noam Sobel, Department of Neurobiology, The Weizmann Institute of Science, Rehovot 76100, Israel, E-mail: lee.sela@weizmann.ac.il or noam.sobel@weizmann.ac.il. DOl:10.1523/JNEUROSCI.2114-09.2009

Copyright $\odot 2009$ Society for Neuroscience $\quad$ 0270-6474/09/2912059-11\$15.00/0
}

(Slotnick and Risser, 1990; McBride and Slotnick, 1997; Kawagoe et al., 2007). These findings have generated the view that, in olfaction, the thalamus may subserve higher-order processing (Eichenbaum et al., 1980; Slotnick and Kaneko, 1981; Slotnick and Risser, 1990). Consistent with this view, single-patient case studies suggested that thalamic lesions may spare detection but impair both discrimination (Potter and Butters, 1980) and hedonic judgments of odors (Rousseaux et al., 1996; Asai et al., 2008). In turn, a comprehensive brain imaging study found a thalamic role in olfactory attention (Plailly et al., 2008). However, there remains no inclusive basic-processing characterization of human olfaction after thalamic lesions. The scope of such a characterization is doubled in light asymmetries in thalamic structural (Eidelberg and Galaburda, 1982; Amunts, 2008), biochemical (Jayasundar, 2002), and functional (Ojemann and Van Buren, 1967; Ojemann, 1977; Mateer, 1978; Schuurman et al., 2002; Whelan et al., 2002; Marchetti et al., 2005) processing.

With all this in mind, we set out to assess the role of the thalamus in olfaction by comparing olfactory processing in patients with thalamic lesions and healthy age-matched controls. In view of thalamic asymmetry, combined with the primarily unilateral and ipsilateral connectivity in olfaction (Price and Slotnick, 1983), we recruited patients with unilateral lesions and conducted all tests twice, once in each nostril. We tested basic olfactory functions, such as detection, as well as higher-order olfactory functioning, such as odor identification. Finally, to directly probe the anecdotal reports of shifts in odorant pleasantness after thalamic lesions, we included an odorant pleasantness estimation task, as well as an auditory control, so as to address whether any anhedonia was modality specific. 


\section{Materials and Methods}

Subjects

All participants (patients and controls) provided informed consent to procedures approved by the Committee for Protection of Human Subjects at the Loewenstein Rehabilitation Hospital.

\section{Patients}

Seventeen patients (three females; mean age, 60.6 years; range, $44-68$ years) with unilateral focal thalamic lesions resulting from cerebral hemorrhage (11 patients), ischemic infarctions (five patients), or abscess (one patient) participated in the study. The patients were current $(n=3)$ and past $(n=14)$ patients of the Loewenstein Rehabilitation Hospital. Nine patients had a lesion of the right thalamus, and eight patients had a lesion of the left thalamus (Fig. 1). Two of the eight left thalamic lesioned patients (P16 and P17) had evidence of some right thalamic damage as well. Eight of the patients had additional extrathalamic damage; two patients (P1 and P3) had left periventricular white-matter lacunar infarctions, three patients (P6, P8, and P9) had minute capsolarputaminal lacunar infarctions, patient $\mathrm{P} 12$ had bilateral lingual and parahippocampal gyri infarctions, patient P17 had mild to moderate enlargement of the ventricular system, and patient P16 had a hypodense lesion close to the anterior right lateral ventricle. Mean time from lesion was $33.6 \pm 27.42$ months. None of the patients had a history of nasal insults or surgery. One patient was an active smoker, and five patients quit smoking $>25$ years earlier.

All patients underwent an assessment of cognitive functioning using the Loewenstein Occupational Therapy Cognitive Assessment (LOTCA) [this battery contains subtests for orientation, visual perception, spatial perception, praxis, visuomotor integration, numerical calculation capacity, and basic thought processes, such as classification and problem solving (Katz et al., 1989)] and the cognitive part of the Functional Independence Measure (FIM-C) [five items (expressive communication, comprehension, memory, social interaction, and problem solving) are scored on a 1-7 point scale (Hamilton et al., 1994)]. In all cases suspected to have spatial neglect, patients were tested with the Behavioral Inattention Test (BIT) [a standardized test battery for neglect comprising cancellation, line bisection, copying, and drawing tasks (Wilson et al., 1989; Hartman-Maeir and Katz, 1995)].

Regarding cognitive state, none of the patients was impaired on the above cognitive tests at a level that could prevent comprehension and full compliance with the instructions of this study or production of coherent and reliable verbal responses (mean LOTCA, $86 \pm 7.8 \%$ correct; mean FIM, $32.66 \pm 3.66$ of 35 ). To further address a possible influence of any memory impairments on olfactory identification, FIM memory scores were correlated with olfactory identification scores. Regarding neglect, only one patient (P5) scored at the cutoff level for neglect (129 of 146 points), with the remaining patients scoring well clear of neglect (mean score, $142.5 \pm 2.65)$. Neglect status was also later considered as a possible factor in olfactory performance.

At interview, most patients did not report alterations in smell or taste after their lesion. However, patients P14 and P2 (9 and 48 months after lesion, respectively) described a persistent postlesion reduction in their awareness of odors, and patient P5 (49 months after lesion) described a persistent reduction in awareness of only unpleasant odors. Patient P10 (17 months after lesion) reported postlesion anosmia that had subsided by the time of the study. In addition, patients $\mathrm{P} 2$ and P10 also reported food anhedonia, and P14 reported sensing bitter taste after eating $(48,17$, and 9 months after lesion, respectively). An additional patient, P15 (25 months after lesion) reported altered taste preferences without olfactory alterations that lasted for 18 months after lesion.

\section{Lesion localization}

Lesion localization within the thalamus was made by review of follow-up brain computed tomography (CT) scans performed $4-8$ weeks after the onset of brain damage. Lesions were localized to the MD, pulvinar, or lateral-compartment subregions of the thalamus (Table 1) according to standard anatomical markers (DeArmond et al., 1976; Talairach and Tournoux, 1988) and reported case studies (Schmahmann, 2003; Carrera and Bogousslavsky, 2006).

\section{Lesion volume quantification}

Lesion volume was measured for each subject based on CT scans digitized and analyzed with the public domain NIH Image software package (http://rsb.info.nih.gov/nih-image/). Lesion volumes were compared with the thalamic volume in the healthy hemisphere to quantify the percentage of thalamus lesioned. In the two cases of bilateral lesions, lesion volume was compared with the less damaged hemisphere.

\section{Healthy controls}

Eighteen healthy controls matched for age (mean age, 60.8 years; range, $47-72$ years; difference from patients, $\left.t_{(33)}=0.131 ; p<0.89\right)$ and gender (four females) participated in the study (Table 1). Seven control subjects 
Table 1. Patients with thalamic lesions and healthy age-matched controls

\begin{tabular}{|c|c|c|c|c|c|c|c|}
\hline & Gender & Age & Education (years) & Lesion classification & Lesion side & Time after onset (months) & Lesion localization \\
\hline \multicolumn{8}{|l|}{ Patient } \\
\hline P1 & Male & 60 & 15 & $\mathrm{H}$ & Right & 40 & Lateral compartment \\
\hline $\mathrm{P} 2$ & Male & 56 & 16 & $\mathrm{H}$ & Right & 48 & MD, lateral compartment \\
\hline P3 & Male & 65 & 0 & $\mathrm{H}$ & Right & 8 & MD, pulvinar, lateral compartment \\
\hline P4 & Female & 60 & 12 & $\mathrm{H}$ & Right & 102 & MD, lateral compartment \\
\hline P5 & Male & 63 & 14 & $\mathrm{H}$ & Right & 49 & Lateral compartment \\
\hline P6 & Male & 56 & 12 & $\mathrm{H}$ & Right & 38 & Lateral compartment \\
\hline P7 & Male & 64 & 17 & $\mathrm{H}$ & Right & 74 & MD, pulvinar, lateral compartment \\
\hline P8 & Male & 57 & 12 & I & Right & Unknown $^{b}$ & MD \\
\hline P9 & Male & 56 & 8 & I & Right & 63 & MD, lateral compartment \\
\hline P10 & Female & 68 & 12 & $\mathrm{H}$ & Left & 17 & Lateral compartment \\
\hline P11 & Male & 62 & 8 & $\mathrm{H}$ & Left & 32 & MD, pulvinar, lateral compartment \\
\hline P12 & Male & 63 & 8 & I & Left & 1 & Lateral compartment \\
\hline P13 & Male & 65 & 12 & $\mathrm{H}$ & Left & 30 & Lateral compartment \\
\hline P14 & Male & 62 & 12 & I & Left & 9 & MD, lateral compartment \\
\hline P15 & Male & 66 & 15 & I & Left & 25 & Lateral compartment \\
\hline P16 & Female & 44 & 12 & $A$ & Left $^{a}$ & 28 & Left MD, right lateral compartment \\
\hline P17 & Male & 63 & 11 & $\mathrm{H}$ & Left $^{a}$ & 3 & Left lateral compartment, right pulvinar \\
\hline \multicolumn{8}{|c|}{ Healthy control } \\
\hline C1 & Male & 60 & 12 & & & & \\
\hline $\mathrm{C} 2$ & Male & 59 & 16 & & & & \\
\hline C 3 & Male & 64 & 12 & & & & \\
\hline C4 & Female & 60 & 17 & & & & \\
\hline $\mathrm{C} 5$ & Male & 61 & 12 & & & & \\
\hline C6 & Male & 54 & 12 & & & & \\
\hline $\mathrm{C7}$ & Male & 62 & 19 & & & & \\
\hline C8 & Male & 61 & 14 & & & & \\
\hline C9 & Male & 56 & 16 & & & & \\
\hline $\mathrm{C} 10$ & Female & 72 & 12 & & & & \\
\hline C11 & Male & 66 & 8 & & & & \\
\hline $\mathrm{C} 12$ & Male & 61 & 12 & & & & \\
\hline $\mathrm{C} 13$ & Female & 59 & 13 & & & & \\
\hline C14 & Male & 61 & 15 & & & & \\
\hline $\mathrm{C} 15$ & Male & 69 & 11 & & & & \\
\hline $\mathrm{C} 16$ & Female & 47 & 18 & & & & \\
\hline C17 & Male & 61 & 17 & & & & \\
\hline C18 & Male & 62 & 12 & & & & \\
\hline
\end{tabular}

H, Hemorrhage; I, ischemic infarction; A, abscess.

${ }^{a}$ There was evidence of some right thalamic damage as well.

${ }^{b}$ The patient was unaware of the lesion that was identified retroactively after the occurrence of a later ischemic infarction.

were active smokers, and four had quit smoking $>10$ years earlier. One control subject had septoplasty 20 years earlier, and no other subject had a history of nasal insult or surgery.

For both experimental order and analyses purposes, each healthy control served as a designated match for a given patient (based on best age and gender match). Thus, for example, if the right nostril of a patient was ipsilesional and was tested before the left nostril, then the right nostril of the matching control was designated "ipsilesional" and was similarly tested before the left nostril.

\section{Procedure}

The experiments took place at the Loewenstein Rehabilitation Hospital or at the subject's own house (control subjects were correspondingly tested at the hospital or their own home, such that testing location would not underlie group differences). Every subject participated in the following tasks: olfactory detection, olfactory identification, and olfactory as well as auditory pleasantness and intensity scoring. The order of the tasks was maintained across subjects, and the whole experiment lasted $\sim 3.5 \mathrm{~h}$. The detection task was the first task administered, so as to measure subjects' thresholds before participating in other olfactory tasks. Whereas olfactory detection and identification tasks were completed first in one nostril and then in the other (counterbalanced for order), pleasantness and intensity scoring tasks were tested by alternating across nostrils every trial. Therefore, the nostril occlusion method differed between tasks. Whereas in the former tasks we used odorless microfoam tape cut to fit over the borders of the naris (Doty, 1992), in the latter tasks we taught the subjects to block their nostril by pressing on the alar (outer lateral) aspect of the nose (Sobel et al., 1999). A computerized interface controlled the experiment [Psyscope software (Cohen et al., 1993)], and all instructions and interaction with subjects were through computer-generated digitized voice. For example, at every trial, the computer generated the following voice command: "please prepare to sniff at the tone, three, two, one, tone." All tasks required the subject's response. Because some of the patients had motor difficulties, all subjects answered verbally, and the experimenter generated the relevant keyboard press. This method prevented motor errors that may mistakenly be attributed to sensory or cognitive disabilities. There was no maximal limitation on response time. The interstimulus interval (ISI) was $25 \mathrm{~s}$ for olfactory tasks and $15 \mathrm{~s}$ for auditory tasks. Every trial ended after the subject's oral response or the minimal ISI, whichever came first.

\section{Tasks}

Olfactory detection thresholds. Detection thresholds were measured for the odorant phenethyl alcohol (PEA) (CAS \#60-12-8) using the signaldetection theory-based "adaptive staircase method" (Cornsweet, 1962). We used 30 dilutions of the odorant PEA, with the highest concentration set at $50 \% \mathrm{v} / \mathrm{v}$ in mineral oil. Participants were blindfolded for the purpose of the task. On each trial, two plastic jars were presented to the subject, one after the other. One jar contained a given concentration of the odorant PEA dissolved in mineral oil, and the other jar (blank) con- 
tained mineral oil alone. On every trial, the subject had to decide which jar contained a higher concentration of odor (forced choice). After the subject's response, computerized feedback was transmitted regarding the success in the current trial.

In the adaptive staircase method, the session began with an average concentration of $-10 \log$ concentrations and continued by a 1-up-2down rule, as follows: five successful consecutive trials with the starting concentration resulted in a reduction of stimulus concentration in the following trial. Two successful trials with a given concentration led to additional concentration reduction. Any failure to detect an odor led to increase in stimulus concentration in the next trial. A failure to detect an odor and a subsequent success with the successive higher concentration were considered as one block. The session stopped after the end of three blocks. The threshold was determined as the average of the four last concentrations in the session. Using this task, we set the threshold of one nostril after the other. Nostril order was counterbalanced across subjects and was identical for a patient and their matching healthy control.

Olfactory identification test. Olfactory identification was assessed by using the standardized University of Pennsylvania Smell Identification Test (UPSIT) (Doty et al., 1984). The UPSIT consists of 40 fouralternative forced-choice items. Each item consists of 10-50 $\mu \mathrm{m}$ ureaformaldehyde polymer microencapsules fixed in proprietary binder and positioned on a brown strip at the bottom of the test booklet. The experimenter released the odorant by scratching the strip with a special pencil tip in a standardized manner. Each strip was incorporated with a multiple-choice question with four alternative answers. After smelling the odorant, the subject could read the answer choices from a computer monitor, and these were also read to them aloud. Furthermore, to overcome any possible effect of reading disability or verbal comprehension, we added a representative picture for each possible answer. The subject was then asked to decide which of the items was the most similar to the smelled odorant. We scored the UPSIT by the proportion of correct answers of 20 items for each nostril, a method commonly used for unilateral testing (Doty, 1992; Good et al., 2003). The order of nostrils and items was counterbalanced across subjects and was identical for a patient and their matching healthy control.

Olfactory and auditory pleasantness and intensity scoring. Olfactory hedonic perception was estimated by using three pleasant, three intermediate, and three unpleasant odorants (Khan et al., 2007), as well as an odorless stimulus. The odorants were as follows: unpleasant included heptanal (CAS \#111-71-7), guaiacol (CAS \#90-05-1), and hexanoic acid (CAS \#142-62-1); intermediate included acetophenone (CAS \#98-86-2), phenethyl alcohol (CAS \#60-12-8), and eugenol (CAS \#97-53-0); and pleasant included ethyl butyrate (CAS \#105-54-4), diphenyl oxide (CAS \#101-84-8), and amyl acetate (CAS \#123-92-2) and the odorless mineral oil alone (for comment about hedonic ordering of the odor phenethyl alcohol, see Results). The odorants were first individually diluted such that they were equated for intensity in a separate group of young adults.

To ask whether thalamic lesions lead to anhedonia specific to olfaction or a more general phenomenon that includes other senses as well, we used audition as a control modality. For auditory hedonic perception, we used recordings of everyday sounds with norms for pleasantness (Marcell et al., 2000). We calculated the distances between pleasantness ratings of the different selected odors from Khan et al. (2007) and matched those distances to the sound pleasantness scale of Marcell et al. (2000) to select three pleasant, three intermediate, and three unpleasant sounds. The sounds were as follows: unpleasant included chewing, zipper, and drill; intermediate included jackhammer, whistling, and river; and pleasant included organ, guitar, and accordion (for comment about hedonic ordering, see Results). A period of quiet (no sound generated) was used as blank. Subjects were instructed that, during the task, they would encounter trials of odor and sound stimuli alternately. The odorants were delivered via plastic jars and the sounds through headphones.

During odor trials, subjects were instructed to take one vigorous sniff, and, during sound trials, they were required to listen. After the stimulus presentation, subjects were asked to rate the stimulus pleasantness and intensity. The ratings were made verbally on a 5-point scale that was incorporated with category names and symbolic pictures. Thus, the pleasantness scale ranged from 1 ("very unpleasant") to 5 ("very pleas- ant"), and the equivalent smiley pictures ranged from a sad smiley icon to a happy smiley icon. Similarly, the intensity scale ranged from 1 ("very weak") to 5 ("very strong"), and the accompanied pictures ranged from a small light blue circle to a large dark blue circle.

Odorants were delivered to each nostril separately (the other blocked by alar pressure). At the beginning of every odor trial, there was an auditory and visual signal that indicated to the subject which nostril to block. For example, in left nostril trials, the note was "please occlude your right nostril." Using this method of occlusion, we could randomize the order of odors and nostrils jointly. Auditory stimuli were similarly delivered to one ear at a time. All stimuli were presented in a randomized order of modality, stimulus, and stimulated side.

Olfactomotor function. Real-time airflow was measured concurrent with the olfactory tasks by nestling a tiny $(<2 \mathrm{~mm})$ tube at the opening of the nostrils (REF 4804*; Salter Labs) (for methods in detail, see Johnson and Sobel, 2007). The tube was attached to a spirometer (ML140; ADInstruments) in which pressure differences were converted into a voltage that was proportional to airflow. The signal was amplified and digitally recorded at $1000 \mathrm{~Hz}$ (ML870; ADInstruments). Sniffs were processed by removing baseline offsets and aligned in time by setting the point at which the sniff passed from the expiratory phase to the inspiratory phase as time 0 . Sniff offset was set to the point at which the sniff returned to zero flow. Sniff volume was calculated by the trapezoidal Reimann sum method. For analysis of sniff airflow, values from left and right nostrils were averaged to one value. This is because, unlike the olfactory sensory signal that feeds separate left and right neural subsystems, the olfactory motor act (sniff) is the result of one centralized neural mechanism, primarily the driver of the intercostal muscles. The differences in airflow between left and right nostrils are a reflection of asymmetric nasal obstruction by swelling of the medial turbinate (Principato and Ozenberger, 1970; Hasegawa and Kern, 1977) and are therefore not of interest in the context of this study.

\section{Exclusions}

One candidate healthy control subject was excluded after failure to detect the strongest concentration of the odor in the detection test, and an alternative healthy control was recruited. For analysis, an exclusion criterion consisting of behavioral performance that deviated by more than 2 SDs from the group average was applied. This criterion led to no exclusions in the detection task, one patient in the identification task, one control subject in each of pleasantness and intensity scoring task, and one patient in the analysis of correlations between lesion volume and behavior. In the olfactomotor function analysis, three subjects were excluded from detection analysis because of insufficient number of recording trials, and two subjects were excluded from pleasantness analysis as a result of failed nostril blocking. The exclusion criteria we applied were consistent with Peirce's criterion for outlier selection (Ross, 2003). In addition, for every result, we also reported the outcome with inclusion of outliers.

\section{Results}

\section{Thalamic lesions did not significantly influence olfactory detection}

A repeated-measures ANOVA on detection thresholds with factors of group (patients and controls), side of lesion (right and left), and stimulated side (ipsilesional and contralesional nostril) revealed a significant interaction of lesion side and stimulated nostril $\left(F_{(1,30)}=6.04, p<0.02\right)$. However, there was no significant difference in detection thresholds between patients and controls (mean patients, -15.34 log concentration; mean controls, $-18.77 \log$ concentration; $\left.F_{(1,30)}=1.61, p<0.2\right)$, nor across nostrils of thalamic patients (mean ipsilesional, $-14.48 \mathrm{log}$ concentration; mean contralesional, -16.2 log concentration; $F_{(1,30)}=2.46, p<0.12$ ) or healthy controls (mean ipsilesional, $-19.29 \log$ concentration; mean contralesional, -18.28 log con- 


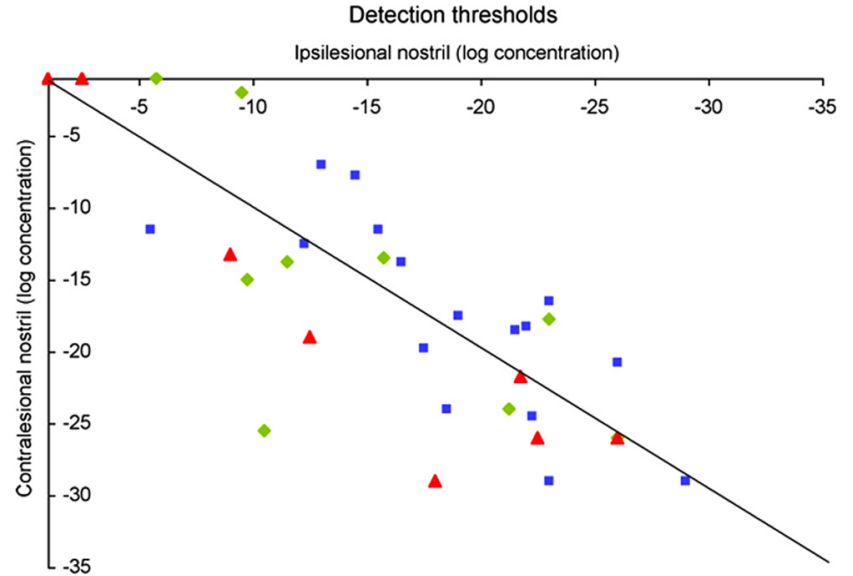

$\diamond$ Right thalamic lesioned patients $=$ Healthy controls $\Delta$ Left thalamic lesioned patients

Figure 2. Odor detection thresholds. Each symbol represents results from one participant: a right thalamic lesioned patient $(\diamond)$, a left thalamic lesioned patient $(\triangle)$, or a healthy control subject $(\square)$. The diagonal line is the unit slope line. Symbols distributed uniformly around this line would correspond to identical performance with the ipsilesional and contralesional nostrils; symbols above the line correspond to better performance with the ipsilesional nostril. Higher negative values indicate better performance. There was no difference in detection thresholds between patients and controls or across nostrils of each group.
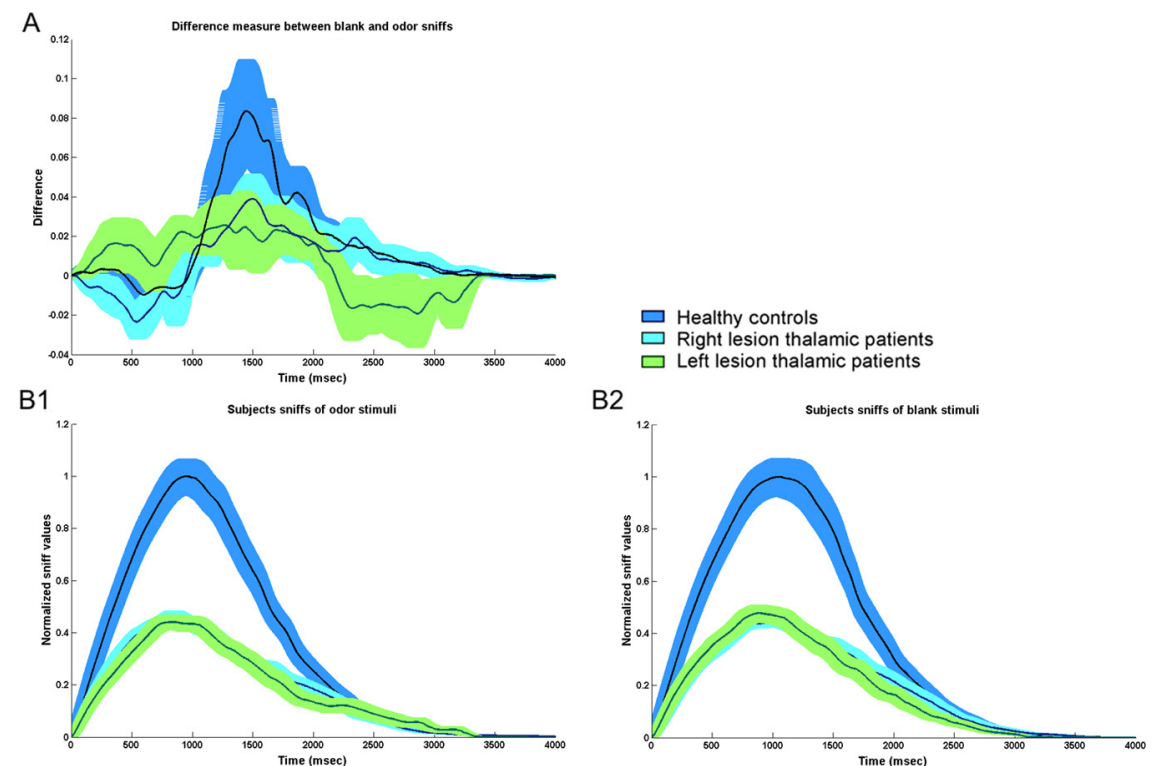

Figure 3. Sniff response of patients and healthy controls during odor detection. $A$, Difference measurement between non-odor and odor sniffs. Means and SE bars are plotted (blue, control group; cyan, right thalamic lesioned patients; green, left thalamic lesioned patients). The difference between odor and non-odor sniffs was significant among healthy controls and right thalamic patients only. Left thalamic lesioned patients did not modulate their sniff in relation to odorant content. $\boldsymbol{B}$, Left and right thalamic lesioned patients took sniffs that were similar in duration but significantly reduced in volume compared with healthy controls. Normalized means and raw SE bars are plotted. B1, Sniffs of non-odor stimuli; $B$ 2, sniffs of odor stimuli.

centration; $\left.F_{(1,30)}=0.55, p<0.46\right)$. Planned contrasts did not reveal significant differences in all cases (Fig. 2).

\section{Thalamic lesions influenced olfactomotor function during olfactory detection}

The following sniff parameters were analyzed: sniff duration, sniff airflow mean velocity, sniff airflow maximum velocity, and sniff volume. Because the parameters of mean sniff velocity, max- imal sniff velocity, and sniff volume were highly correlated (all $r>0.7$, all $p<0.006$ ), for the sake of brevity, we will present sniff duration and sniff volume.

\section{Sniff duration}

A repeated-measures ANOVA on sniff duration with factors of group (patients and controls), side of lesion (right and left), and stimulus type (blank and odor) revealed a significant effect of stimulus type (mean blank, $1.96 \pm 0.44 \mathrm{~s}$; mean odor, $1.87 \pm$ 0.43 s; $\left.F_{(1,28)}=15.22, p<0.0005\right)$. Planned contrasts revealed that subjects in the control group took significantly longer sniffs to a blank olfactory stimulus compared with an odorant stimulus (mean blank, $2 \pm 0.42 \mathrm{~s}$; mean odor, $1.92 \pm 0.4 \mathrm{~s} ; F_{(1,28)}=9.94$, $p<0.0038)$. However, whereas right thalamic lesioned patients also took longer sniffs of blank than of odorant (mean blank, $2.08 \pm 0.45 \mathrm{~s}$; mean odor, $1.94 \pm 0.44 \mathrm{~s} ; F_{(1,28)}=9.13$, $p<0.005)$, left thalamic lesioned patients failed to do so (mean blank, $1.73 \pm 0.41 \mathrm{~s}$; mean odor, $1.71 \pm 0.51 \mathrm{~s} ; F_{(1,28)}=$ $0.158, p<0.69)$ (Fig. 3A).

Planned contrasts revealed that there was no difference in sniff duration of patients and controls for odor stimuli (mean patients, $1.83 \pm 0.47 \mathrm{~s}$; mean controls, $\left.1.91 \pm 0.4 \mathrm{~s} ; F_{(1,28)}=0.26, p<0.62\right)$ or blank (mean patients, $1.91 \pm 0.45 \mathrm{~s}$; mean controls, $2 \pm 0.43 \mathrm{~s}$; $\left.F_{(1,28)}=0.35, p<0.56\right)$. There was no difference in sniff duration of left and right thalamic lesioned patients (mean left, $1.72 \pm$ $0.44 \mathrm{~s}$; mean right, $2.01 \pm 0.44 \mathrm{~s} ; F_{(1,28)}=$ $1.55, p<0.22)$ (Fig. 3B).

\section{Sniff volume}

A repeated-measures ANOVA on sniff volume with factors of group (patients and controls), side of lesion (right and left), and stimulus type (blank and odor) revealed significant effects of group (mean patients, $111.94 \pm 47.39 \mathrm{ml}$; mean controls, $218.68 \pm 130.07 \mathrm{ml} ; F_{(1,28)}=$ $7.71, p<0.009$ ) and stimulus type (mean blank, $178.88 \pm 123.1 \mathrm{ml}$; mean odor, $165.08 \pm 107.67 \mathrm{ml} ; F_{(1,28)}=10.24, p<$ $0.003)$. Planned contrasts revealed that, whereas control subjects took greater sniffs of blank stimulus compared with an odor (mean blank, $228.01 \pm 146.95 \mathrm{ml}$; mean odor, $209.35 \pm 47.04 \mathrm{ml}$; $F_{(1,28)}=$ $11.65, p<0.002)$, this effect was absent in left and right lesioned patient groups (mean left blank, $111.09 \pm 53.86 \mathrm{ml}$; mean left odor, $105.84 \pm 54.24 \mathrm{ml}$; $F_{(1,28)}=0.50, p<0.48 ;$ mean right blank, $119.52 \pm 47.29 \mathrm{ml}$; mean right odor, $110.47 \pm 43.47 \mathrm{ml} ; F_{(1,28)}=1.11$, $p<0.29)$ (Fig. 3A).

Planned contrasts revealed that sniff volume of patients was smaller than controls for odor stimuli (mean patients, $108.16 \pm 47.4 \mathrm{ml}$; mean controls, $209.35 \pm 121.16 \mathrm{ml} ; F_{(1,28)}=8$, $p<0.008$ ) and blank (mean patients, $115.71 \pm 48.85 \mathrm{ml}$; mean controls, $\left.228.01 \pm 141.32 \mathrm{ml} ; F_{(1,28)}=7.39, p<0.011\right)$. There was no difference between sniff volumes of left and right thalamic lesioned patients (mean left, $108.87 \pm 52.03 \mathrm{ml}$; mean right, $\left.115 \pm 44.01 \mathrm{ml} ; F_{(1,28)}=0.011, p<0.91\right)$ (Fig. 3B).

In other words, left thalamic lesions influenced olfactomotor modulation but did not influence detection thresholds. 


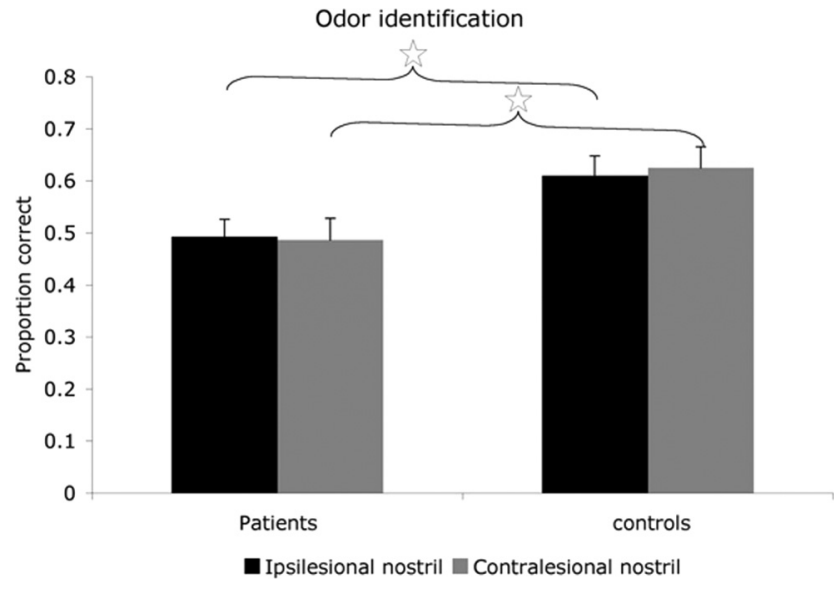

Figure 4. Odor identification. Mean proportion of correct answers using each nostril is plotted. Error bars are SE; $₫ p<0.03$. Note that patients were impaired at identifying odors relative to controls with both nostrils.

\section{Thalamic lesions impaired olfactory identification}

A repeated-measures ANOVA on the proportion of correct answers within each nostril with factors of group (patients and controls), side of lesion (right and left), and stimulated side (ipsilesional and contralesional nostril) revealed a significant main effect of group $\left(F_{(1,28)}=8.05, p<0.008\right)$. Planned contrasts revealed that patients identified significantly fewer odors compared with controls in both nostrils (mean ipsilesional patients, $0.49 \pm 0.13$; mean ipsilesional controls, $0.61 \pm 0.15 ; F_{(1,28)}=$ 5.47, $p<0.03$; mean contralesional patients, $0.49 \pm 0.17$; mean contralesional controls, $0.63 \pm 0.16 ; F_{(1,28)}=5.57, p<0.03$ ) (Fig. $4)$. Moreover, planned contrasts revealed a significant impairment in right thalamic lesioned patients compared with their matched controls (mean right patients, $0.46 \pm 0.15$; mean right controls, $\left.0.65 \pm 0.10 ; F_{(1,28)}=8.69, p<0.006\right)$ but not in left thalamic lesioned patients compared with their controls (mean left patients, $0.51 \pm 0.07$; mean left controls, $0.59 \pm 0.16 ; F_{(1,28)}=$ $1.13, p<0.29)$. None of the groups had a difference in identification rates between the nostrils (mean patients ipsilesional, $0.49 \pm 0.14$; mean patients contralesional, $0.49 \pm 0.17 ; F_{(1,28)}=$ 0.024, $p<0.87$; mean controls ipsilesional, $0.61 \pm 0.15$; mean controls contralesional, $\left.0.63 \pm 0.16 ; F_{(1,28)}=0.12, p<0.74\right)$. Repeating the analysis after including the outlier patient did not change results. Finally, memory scores were unrelated to performance in olfactory identification (all $r<0.1$; all $p>$ 0.72 ), yet patient P5, who reached the cutoff score for neglect, was significantly impaired compared with the patient mean $(Z=2.86, p<0.004)$.

\section{Right thalamic lesions reduced odorant but not sound pleasantness}

The hedonic ordering of both odors and sounds in the agematched healthy controls was slightly different from the expected in healthy young subjects (for sounds, Marcell et al., 2000; for odors, Khan et al., 2007). Specifically, one pair of stimuli substituted positions in the odor domain (the odors ethyl butyrate and phenethyl alcohol) and two pairs in the sound domain (the sounds river and chewing with the sounds organ and jackhammer, respectively). Here we opted to compare the patients with the age-matched healthy controls rather than with the slightly different expectation from young controls tested by others elsewhere.

A repeated-measures ANOVA for odors with factors of group (patients and controls), side of lesion (right and left), stimulus valence (pleasant, intermediate, and unpleasant), and stimulated side (ipsilesional and contralesional nostril) revealed a significant main effect of stimulus valence $\left(F_{(2,58)}=37.99, p<0.0001\right)$ and significant interactions of group and stimulus valence $\left(F_{(2,58)}=\right.$ 5.06, $p<0.009)$, stimulus valence and stimulated nostril $\left(F_{(2,58)}=\right.$ $4.18, p<0.02)$, and group, lesion side, and stimulus valence $\left(F_{(2,58)}=3.69, p<0.031\right)$. A similar analysis for sounds revealed a significant effect for stimulus valence only $\left(F_{(2,58)}=158.01, p<\right.$ $0.0001)$. Repeating the analysis after including the outlier control subject did not change results.

\section{Differences in hedonic ratings of pleasant and unpleasant stimuli}

Planned contrasts revealed that controls rated pleasant odorants as significantly more pleasant than the unpleasant odorants in both nostrils (mean ipsilesional pleasant, $3.29 \pm 0.77$; mean ipsilesional unpleasant, $2.08 \pm 0.56 ; F_{(1,29)}=36.38, p<0.0001$; mean contralesional pleasant, $3.3 \pm 0.64$; mean contralesional unpleasant, $\left.2.05 \pm 0.53 ; F_{(1,29)}=37.27, p<0.0001\right)$ and similarly rated pleasant and unpleasant sounds in both ears (mean ipsilesional pleasant, $4.29 \pm 0.71$; mean ipsilesional unpleasant, $1.66 \pm 0.65 ; F_{(1,29)}=125.37, p<0.0001$; mean contralesional pleasant, $4.27 \pm 0.74$; mean contralesional unpleasant, $1.67 \pm$ $\left.0.61 ; F_{(1,29)}=106.31, p<0.0001\right)$. Left thalamic lesioned patients also rated pleasant odorants as more pleasant than unpleasant odorants in both nostrils (mean ipsilesional pleasant, $3.12 \pm$ 1.01; mean ipsilesional unpleasant, $2.45 \pm 1.28 ; F_{(1,29)}=6.06$, $p<0.019$; mean contralesional pleasant, $3.11 \pm 1$; mean contralesional unpleasant, $2.16 \pm 0.99 ; F_{(1,29)}=11.48, p<0.002$ ) and similarly rated pleasant and unpleasant sounds in both ears (mean ipsilesional pleasant, $3.85 \pm 0.63$; mean ipsilesional unpleasant, $1.85 \pm 0.94 ; F_{(1,29)}=37.66, p<0.0001$; mean contralesional pleasant, $4.08 \pm 0.42$; mean contralesional unpleasant, $\left.1.98 \pm 0.96 ; F_{(1,29)}=36.35, p<0.0001\right)$. However, right thalamic lesioned patients had no difference in their pleasantness ratings of pleasant and unpleasant odorants in both nostrils (mean ipsilesional pleasant, $2.77 \pm 0.95$; mean ipsilesional unpleasant, $2.38 \pm$ $0.83 ; F_{(1,29)}=2.35, p<0.14$; mean contralesional pleasant, $2.46 \pm 0.88$; mean contralesional unpleasant, $2 \pm 0.71 ; F_{(1,29)}=$ $3.09, p<0.09$ ), yet they did rate pleasant sounds as significantly more pleasant than unpleasant sounds in both ears (mean ipsilesional pleasant, $4.35 \pm 0.64$; mean ipsilesional unpleasant, $2 \pm$ $0.5 ; F_{(1,29)}=58.35, p<0.0001$; mean contralesional pleasant, $4.09 \pm 1.16$; mean contralesional unpleasant, $2.15 \pm 0.65 ; F_{(1,29)}=$ 35.06, $p<0.0001$ ) (Fig. 5).

\section{Differences in hedonic ratings across nostrils}

Planned contrasts revealed that right thalamic lesioned patients rated pleasant odorants as less pleasant in the contralesional nostril compared with the ipsilesional nostril (mean ipsilesional, $2.78 \pm 0.95$; mean contralesional, $2.46 \pm 0.88 ; F_{(1,8)}=15.21, p<$ 0.0045 ) and to the matched controls contralesional nostril (mean patients contralesional, $2.46 \pm 0.88$; mean controls contralesional, $\left.3.48 \pm 0.66 ; F_{(1,16)}=7.67, p<0.013\right)$. However, an identical analysis on sounds revealed that there was no difference in the rating of pleasant sounds in right thalamic lesioned patients across ears (mean ipsilesional, $4.35 \pm 0.65$; mean contralesional, $\left.4.09 \pm 1.16 ; F_{(1,29)}=0.09, p<0.76\right)$ or compared with controls (right pleasant, $4.22 \pm 0.92$; controls pleasant, $4.44 \pm 0.59 ; F_{(1,29)}=0.48, p<0.49$ ) (Fig. 5). 

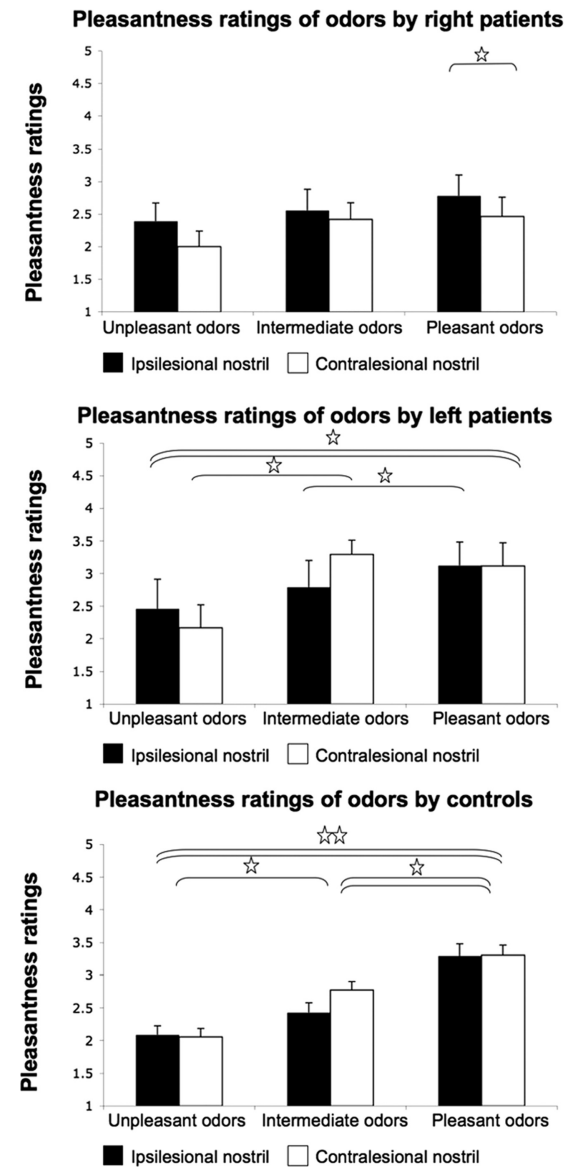

and odor valence (pleasant and unpleasant) revealed a significant effect of odor valence $\left(F_{(1,29)}=5.96, p<0.021\right)$ and a significant interaction between group, lesion side, and odor valence $\left(F_{(1,29)}=\right.$ $6.57, p<0.015)$. Planned contrasts revealed that left thalamic lesioned patients took significantly smaller sniffs to unpleasant olfactory stimuli compared with pleasant olfactory stimuli (mean pleasant, $106.18 \pm 56.73 \mathrm{ml}$; mean unpleasant, $91.65 \pm 59.13 \mathrm{ml} ; F_{(1,29)}=5.52, p<$ 0.025). A similar trend was evident for controls (mean pleasant, $106.5 \pm 57.17$ $\mathrm{ml}$; mean unpleasant, $97.9 \pm 54.78 \mathrm{ml}$; $\left.F_{(1,29)}=3.67, p<0.065\right)$ but no effect was found for right thalamic lesioned patients (mean pleasant, $57.03 \pm 21.13 \mathrm{ml}$; mean unpleasant, $58.07 \pm 27.68 \mathrm{ml} ; F_{(1,29)}=$ $0.03, p<0.86)$ (Fig. 6).

That right thalamic lesioned patients did not modulate their sniff according to odorant hedonics was congruent with their perception that did not discriminate between hedonic categories of odors.

\section{Thalamic lesions did not influence olfactory intensity perception}

A repeated-measures ANOVA with factors of group (patients and controls), side of lesion (right and left), and stimulated side (ipsilesional and contralesional nostril/ear) failed to reveal significant effects of intensity for odor and sound stimuli. There was no difference between intensity scorings of both groups or across nostrils of each group for odors (mean patients ipsilesional, $3.27 \pm 0.62$; mean patients

Figure 5. Pleasantness ratings of olfactory and auditory stimuli. Subjects rated three pleasant, three intermediate, and three unpleasant odorants and sounds on a scale from 1 (very unpleasant) to 5 (very pleasant). Each bar represents the group average valence in one modality within one nostril. Error bars indicates SE. Single horizontal lines represent significant differences in only one nostril/ear, and double lines represent differences for both nostrils/ears. $2 p p<0.03$, 部放 $p<0.0001$. Right thalamic lesioned patients differentiated the pleasantness of sounds but not smells.

\section{Right thalamic lesions influenced olfactomotor function during pleasantness estimation}

As in the case of detection, sniff parameters of mean velocity, maximal velocity, and sniff volume were highly correlated (all $r>$ 0.82; all $p<0.00006$ ), and, therefore, for the sake of brevity, only sniff duration and volume are presented.

\section{Sniff duration}

A repeated-measures ANOVA on sniff duration with factors of group (patients and controls), side of lesion (right and left), and odor valence (pleasant and unpleasant) revealed a significant effect of odor valence $\left(F_{(1,29)}=17.12, p<0.0002\right)$. Planned contrasts revealed that subjects in the control group took significantly shorter sniffs of unpleasant olfactory stimuli compared with pleasant olfactory stimuli (mean pleasant, $2.41 \pm 0.54 \mathrm{~s}$; mean unpleasant, $\left.2.18 \pm 0.54 \mathrm{~s} ; F_{(1,29)}=18.88, p<0.0002\right)$. A similar trend was evident in left thalamic lesioned patients (mean pleasant, $2 \pm 0.44 \mathrm{~s}$; mean unpleasant, $1.85 \pm 0.38 \mathrm{~s} ; F_{(1,29)}=$ $3.89, p<0.058$ ) but not in right thalamic lesioned patients (mean pleasant, $2.17 \pm 0.32 \mathrm{~s}$; mean unpleasant, $2.16 \pm 0.30 \mathrm{~s} ; F_{(1,29)}=$ $0.045, p<0.83$ ) (Fig. 6).

\section{Sniff volume}

A repeated-measures ANOVA on sniff volume with factors of group (patients and controls), side of lesion (right and left), contralesional, $3.38 \pm 0.7$; mean controls ipsilesional, $3.34 \pm 0.6$; mean controls contralesional, $3.38 \pm 0.42$ ) or sounds (mean patients ipsilesional, $3.53 \pm 0.58$; mean patients contralesional, $3.36 \pm 0.62$; mean controls ipsilesional, $3.49 \pm 0.38$; mean controls contralesional, $3.4 \pm 0.43$ ). In an attempt to reveal whether the difference in pleasantness scoring that was found across nostrils among right thalamic patients was a reflection of a similar change in intensity rating, the same contrast was made for intensity. However, planned contrasts failed to reveal a difference in intensity ratings across nostrils in right thalamic patients (mean ipsilesional, $3.17 \pm 0.6$; mean contralesional, $3.48 \pm$ $0.62 ; F_{(1,29)}=2.89, p<0.10$ ) (Fig. 7). Therefore, changes found in pleasantness scoring were not related to changes in intensity perception. Repeating the analysis after including the outlier control subject did not change results.

\section{Pulvinar damage was associated with worse} olfactory performance

To ask whether lesion type (hemorrhage, ischemic infarction, or abscess) or location within the thalamus (MD, lateral compartment, or pulvinar) had different effects on behavioral results or on olfactomotor function, we conducted a Kruskal-Wallis analysis with each of the grouping factors: lesion type and lesion location as independent factors, and results of ipsilesional and contralesional nostrils on every task as dependent factors. 


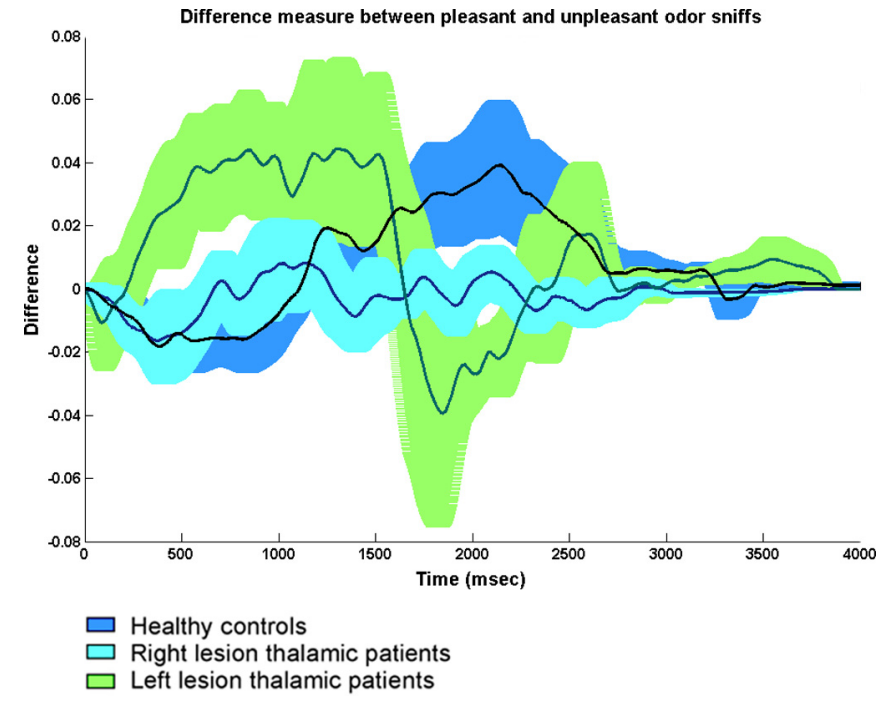

Figure 6. Difference measurement between sniffs for pleasant and unpleasant odorants. Means and SE bars are plotted (blue, control group; cyan, right thalamic lesioned patients; green, left thalamic lesioned patients). Whereas healthy controls and left thalamic patients took longer sniffs of pleasant than unpleasant odorants, right thalamic patients took equal sniffs of pleasant and unpleasant odorants.

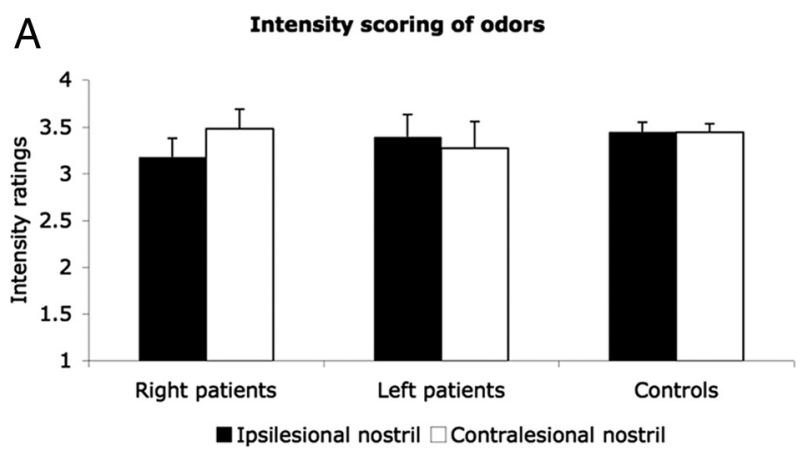

B

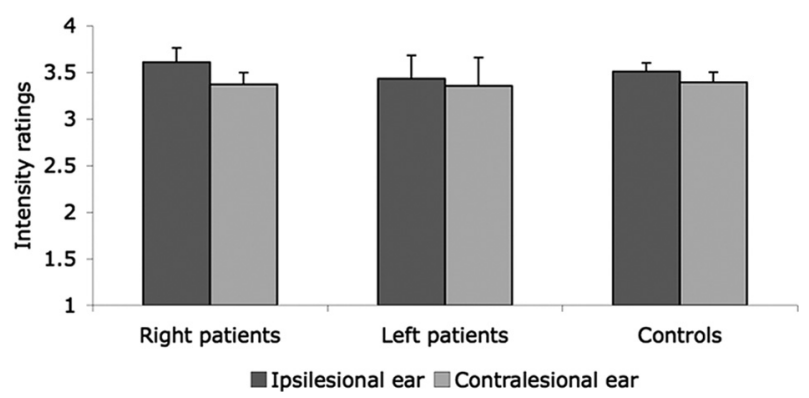

Figure 7. Intensity ratings of olfactory $(\boldsymbol{A})$ and auditory $(\boldsymbol{B})$ stimuli. Subjects rated 10 odorants and sounds on a scale from 1 (very unpleasant) to 5 (very pleasant). Each bar represents the group average of ratings of all stimuli from one modality within one nostril. Error bars indicates SE. There were no significant differences between groups or stimulated side (of nostrils or ears).

Patients with pulvinar lesions performed significantly worse than other patients in all behavioral tasks; in the ipsilesional nostril during detection $\left(H_{(1, n=17)}=5.39, p<0.02\right)$, ipsilesional nostril during identification $\left(H_{(1, n=16)}=3.82, p<0.05\right)$, ipsilesional nostril during pleasantness scoring $\left(H_{(1, n=17)}=5.14, p<\right.$ $0.023)$, and contralesional nostril during pleasantness scoring $\left(H_{(1, n=17)}=6.21, p<0.012\right)$. In addition, lesions in the lateral compartment of the thalamus were associated with better perfor- mance in the ipsilesional nostril during identification $\left(H_{(1, n=16)}=\right.$ $5.39, p<0.02$ ). None of the lesion locations specifically affected olfactomotor function. Moreover, in light of previous studies implicating the MD nucleus in olfaction, we reanalyzed the data contrasting $\mathrm{MD}$ lesions $(n=9)$ with non-MD lesions $(n=8)$ using parametric (ANOVA) rather than nonparametric (Kruskal-Wallis) statistics. This analysis for all the above tasks revealed only one significant third-level interaction in the duration parameter of sniff analysis during odor pleasantness scoring with factors of group (patients and control), lesion location (MD and non-MD), and odor valence (pleasant and unpleasant) $\left(F_{(1,28)}=6.99, p<0.013\right.$; otherwise, all $F<3.16$, all $\left.p>0.09\right)$. Repeating the analysis after including the outlier patient in the identification task did not change results. In other words, we did not find significant differences in olfactory processing as a result of MD lesions versus non-MD lesions. Further dividing the MD patients into left and right lesioned thalami resulted in group sizes that did not allow meaningful statistical analysis.

\section{Greater thalamic lesion volume was associated with poorer olfactory identification}

To ask whether lesion volume was related to performance, we tested for correlations between the lesion volume and behavioral, as well as olfactomotor performance in both nostrils. Significant correlation coefficients were found only for olfactory identification in both nostrils, whereby higher lesion volume was correlated with poorer performance (ipsilesional nostril, $r=-0.517$, $p<0.03$; contralesional nostril, $r=-0.626, p<0.006)$. Reassessing the proportion of lesion volume in the two bilaterally lesioned patients by now dividing the sum of lesion volumes on both sides by the sum of thalamic volume on both sides did not influence this result. Including outliers in the analysis did not influence results in the ipsilesional nostril yet negated the effect in the contralesional nostril.

\section{Discussion}

Nearly every neuroscience textbook in its chapter on olfaction notes that olfaction is unique in that information arrives at cortex without a thalamic relay. Despite this being one of the "textbook facts" of olfaction, the influence of thalamic lesions on human olfaction has not been systematically tested. Here we conducted such a test and found several impairments and alterations in olfactory function.

In the functional hierarchy of olfaction, olfactory detection may be considered the most basic behavior (Savic et al., 2000). Consistent with findings in rodents, we found that patients with thalamic lesions were not significantly impaired at olfactory detection. This held despite altered olfactomotor function in the left patients during this task. In young healthy subjects, higher odorant concentrations are sniffed with reduced vigor compared with lower concentrations (Laing, 1983; Sobel et al., 2001; Walker et al., 2001; Johnson et al., 2003). Such an olfactomotor response was evident here in healthy age-matched controls and to some extent in right hemisphere thalamic patients but not in left hemisphere patients. The altered olfactomotor response after left thalamic lesions combines with previous reports of preferentially left thalamic control over respiratory expiration (Ojemann and Van Buren, 1967) and thalamic involvement in sniffing behavior (Zainos et al., 1984) to suggest left thalamic involvement in olfactomotor control.

The sensitivity of the olfactomotor test is consistent with the use of this measure as a diagnostic sign of olfactory impairment (Frank et al., 2003; Tourbier and Doty, 2007) and with similar 
alterations in olfactomotor processing in diseases such as Parkinson's disease (Sobel et al., 2001) or after lesions to candidate components of the olfactomotor system such as the cerebellum (Mainland et al., 2005). Although sniffing may be considered an inseparable part of the olfactory percept (Kepecs et al., 2006; Mainland and Sobel, 2006; Schoenfeld and Cleland, 2006), it is nevertheless subserved by an independent olfactomotor system (Sobel et al., 1998). Nonetheless, both left and right thalamic damaged patients used significantly reduced sniff vigor than controls, without a considerable influence on detection thresholds. This dissociation between detection threshold and olfactomotor performance suggests that the thalamus may be a component of the olfactomotor network.

Whereas our findings of olfactomotor alterations after thalamic lesion were novel, our findings regarding an impairment in olfactory identification are consistent with an impairment in olfactory discrimination seen in rodents after thalamic lesions (Eichenbaum et al., 1980; Slotnick and Kaneko, 1981; Staubli et al., 1987; Slotnick and Risser, 1990). Furthermore, a key aspect of our study addressed olfactory hedonics. Anecdotal evidence suggested a thalamic role in olfactory hedonics (Rousseaux et al., 1996; Asai et al., 2008). Our results confirmed these observations but went further to reveal that patients with right, but not left, thalamic lesions experienced altered hedonic perception of odors. Furthermore, this alteration reflected a shift in the perception of pleasant but not unpleasant odors or, in other words, olfactory anhedonia. This olfactory anhedonia is clearly depicted in Figure 5 in which the pleasantness ratings of sounds (right column) clearly increased from the expected unpleasant to pleasant (lower values on the left of the graph, higher on the right). In contrast, whereas a similar picture was evident for odors in healthy controls (bottom left panel), it was not evident in odors for right lesioned patients (top left panel) in which odor pleasantness ratings significantly deviated from expectation. This anhedonia was reflected not only in explicit perceptual ratings but also in the olfactomotor response: consistent with results in young healthy subjects (Bensafi et al., 2003, 2007), age-matched controls took longer sniffs of pleasant odorants and shorter sniffs of unpleasant odorants. In contrast, right hemisphere patients failed to modulate their sniff in accordance with odorant valence. This indicates right thalamic involvement in the olfactomotor response processes and raises the possibility of distinct olfactomotor roles for left and right thalamus. This implicit measure further suggested that the hedonic alteration in these patients was not merely a reflection of their capability to report perception but rather a genuine anhedonia. Finally, an auditory control suggested that this anhedonia was specific to olfaction, and intensity estimations revealed that it did not result from alteration in intensity perception.

The laterality of this effect may contribute to the ongoing debate regarding brain lateralization of emotion. In addition to the textbook notion of right hemisphere general specialization in emotion (Kalat, 2003), models considering hemisphere-specific emotions have emerged. These diverge, however, from those proposing right hemisphere specialization in positive valence versus left hemisphere in negative valence (Kobal et al., 1992; Zald and Pardo, 1997; Herz et al., 1999; Anderson et al., 2003) and those proposing the opposite pattern of right hemisphere specialization in negative valence versus left hemisphere in positive valence (Henkin and Levy, 2001; Bensafi et al., 2002). Our results support the former model, indicating that the thalamus is engaged in positive hedonic processing within the right hemisphere. We did not, however, find evidence for negative hedonic processing in the left hemisphere.

These results also add to the growing body of evidence that points away from the classic notion of the olfactory system as both unilateral and ipsilateral only, i.e., right nostril connected to right brain and vise versa. Here, the effects in identification were bilateral, and the effects in hedonic processing were contralateral. Indeed, careful review of some of the anatomical literature (Price and Slotnick, 1983; Shipley and Ennis, 1996), as well as an overwhelming proportion of recent functional results in both rodents (McBride and Slotnick, 1997; Wilson, 1997; Uva and de Curtis, 2005; Cross et al., 2006) and humans (Savic and Gulyas, 2000; Porter et al., 2005) suggest prominent contralateral and ipsilateral connectivity within the olfactory system. In other words, the olfactory system is both ipsilateral and contralateral.

Although our results allowed us to confidently answer our main question (there is an olfactory impairment after thalamic lesion), our study also had several limitations, and we would like to clearly state those here. Whereas human lesion studies add an important aspect to our understanding of brain function, such studies also suffer from inherent drawbacks. The first is typical sample size. Although our study $(n=17)$ is one of the largest thalamic lesion studies we are aware of, it is nevertheless smaller than a typical rodent lesion study. Furthermore, although 9 of the 17 patients were characterized as having MD lesions, allowing a comparison of MD to non-MD lesion outcome (no differences found); once this group was broken down to left (four) and right (five) MD lesions, this number of patients did not allow in-depth statistical analysis. Thus, our ability to attribute impairments to thalamic subregions was limited. A second drawback common to human lesion studies is the lack of female subjects. This imbalance reflected a known gender imbalance with male predominance in cerebrovascular disease (Prencipe et al., 1997; Sudlow and Warlow, 1997). A third inherent drawback of human lesion studies is that the lesions are often not "clean." Here, approximately half of the patients had additional minimal nonthalamic damage. Finally, one drawback of our study was that lesion localization was based on CT rather than magnetic resonance imaging scans. This constrained our accuracy in defining thalamic subregions. Moreover, our lesion volume analysis was based on digitization of these same CT scans. These scans were taken at similar timeframes after lesion but not at similar timeframes before olfactory testing. This raises a concern of differential recovery not reflected in our analysis. That said, post hoc analysis revealed no significant correlation between time elapsed from lesion and olfactory performance. Furthermore, the lesion measurements were conducted "blind" to lesion outcome, and therefore it is unlikely that this process introduced type I error. In other words, we think that the relationship we uncovered between lesion volume and olfactory identification was genuine.

In conclusion, the current results allowed us to make several novel observations. Thalamic lesions clearly impaired olfactory identification and altered olfactory hedonics by reducing the pleasantness of pleasant odors. These impairments were evident in explicit measures of perception and were reflected in altered olfactomotor function, implicating the thalamus in the neural substrates of the olfactomotor loop.

\section{References}

Amunts VV (2008) Individual variability in the structural asymmetry of the dorsomedial nucleus of the thalamus in men and women. Neurosci Behav Physiol 38:715-720. 
Anderson AK, Christoff K, Stappen I, Panitz D, Ghahremani DG, Glover G, Gabrieli JD, Sobel N (2003) Dissociated neural representations of intensity and valence in human olfaction. Nat Neurosci 6:196-202.

Asai H, Udaka F, Hirano M, Ueno S (2008) Odor abnormalities caused by bilateral thalamic infarction. Clin Neurol Neurosurg 110:500-501.

Bensafi M, Rouby C, Farget V, Vigouroux M, Holley A (2002) Asymmetry of pleasant vs. unpleasant odor processing during affective judgment in humans. Neurosci Lett 328:309-313.

Bensafi M, Porter J, Pouliot S, Mainland J, Johnson B, Zelano C, Young N, Bremner E, Aframian D, Khan R, Sobel N (2003) Olfactomotor activity during imagery mimics that during perception. Nat Neurosci 6:1142-1144.

Bensafi M, Sobel N, Khan RM (2007) Hedonic-specific activity in piriform cortex during odor imagery mimics that during odor perception. J Neurophysiol 98:3254-3262.

Carrera E, Bogousslavsky J (2006) The thalamus and behavior-effects of anatomically distinct strokes. Neurology 66:1817-1823.

Cohen JD, MacWhinney B, Flatt M, Provost J (1993) PsyScope: a new graphic interactive environment for designing psychology experiments. Behav Res Methods Instrum Comput 25:257-271.

Cornsweet TN (1962) The staircrase-method in psychophysics. Am J Psychol 75:485-491.

Cross DJ, Flexman JA, Anzai Y, Morrow TJ, Maravilla KR, Minoshima S (2006) In vivo imaging of functional disruption, recovery and alteration in rat olfactory circuitry after lesion. Neuroimage 32:1265-1272.

DeArmond SJ, Fusco MM, Dewey MM (1976) Structure of the human brain: a photographic atlas, Ed 2. New York: Oxford UP.

Doty RL (1992) Diagnostic tests and assessment. J Head Trauma Rehabil $7: 45-64$.

Doty RL, Shaman P, Dann M (1984) Development of the University of Pennsylvania Smell Identification Test: a standardized microencapsulated test of olfactory function. Physiol Behav 32:489-502.

Eichenbaum H, Shedlack KJ, Eckmann KW (1980) Thalamocortical mechanisms in odor-guided behavior. I. Effects of lesions of the mediodorsal thalamic nucleus and frontal cortex on olfactory discrimination in the rat. Brain Behav Evol 17:255-275.

Eidelberg D, Galaburda AM (1982) Symmetry and asymmetry in the human posterior thalamus. I. Cytoarchitectonic analysis in normal persons. Arch Neurol 39:325-332.

Frank RA, Dulay MF, Gesteland RC (2003) Assessment of the Sniff Magnitude Test as a clinical test of olfactory function. Physiol Behav 78:195-204.

Good KP, Martzke JS, Daoud MA, Kopala LC (2003) Unirhinal norms for the University of Pennsylvania Smell Identification Test. Clin Neuropsychol 17:226-234.

Haberly LB, Price JL (1977) The axonal projection patterns of the mitral and tufted cells of the olfactory bulb in the rat. Brain Res 129:152-157.

Hamilton BB, Laughlin JA, Fiedler RC, Granger CV (1994) Interrater reliability of the 7-level functional independence measure (FIM). Scand J Rehabil Med 26:115-119.

Hartman-Maeir A, Katz N (1995) Validity of the Behavioral Inattention Test (BIT): relationships with functional tasks. Am J Occup Ther 49:507-516.

Hasegawa M, Kern EB (1977) The human nasal cycle. Mayo Clin Proc 52:28-34.

Henkin RI, Levy LM (2001) Lateralization of brain activation to imagination and smell of odors using functional magnetic resonance imaging (fMRI): left hemispheric localization of pleasant and right hemispheric localization of unpleasant odors. J Comput Assist Tomogr 25:493-514.

Herz RS, McCall C, Cahill L (1999) Hemispheric lateralization in the processing of odor pleasantness versus odor names. Chem Senses 24:691-695.

Jayasundar R (2002) Human brain: biochemical lateralization in normal subjects. Neurol India 50:267-271.

Johnson BN, Sobel N (2007) Methods for building an olfactometer with known concentration outcomes. J Neurosci Methods 160:231-245.

Johnson BN, Mainland JD, Sobel N (2003) Rapid olfactory processing implicates subcortical control of an olfactomotor system. J Neurophysiol 90:1084-1094.

Kalat JW (2003) Biological psychology, Ed 8. Belmont, CA: Wadsworth Publishing.

Katz N, Itzkovich M, Averbuch S, Elazar B (1989) Loewenstein Occupa- tional Therapy Cognitive Assessment (LOTCA) battery for brain-injured patients: reliability and validity. Am J Occup Ther 43:184-192.

Kawagoe T, Tamura R, Uwano T, Asahi T, Nishijo H, Eifuku S, Ono T (2007) Neural correlates of stimulus-reward association in the rat mediodorsal thalamus. Neuroreport 18:683-688.

Kay LM, Sherman SM (2007) An argument for an olfactory thalamus. Trends Neurosci 30:47-53.

Kepecs A, Uchida N, Mainen ZF (2006) The sniff as a unit of olfactory processing. Chem Senses 31:167-179.

Khan RM, Luk CH, Flinker A, Aggarwal A, Lapid H, Haddad R, Sobel N (2007) Predicting odor pleasantness from odorant structure: pleasantness as a reflection of the physical world. J Neurosci 27:10015-10023.

Kobal G, Hummel T, Van Toller S (1992) Differences in human chemosensory evoked potentials to olfactory and somatosensory chemical stimuli presented to left and right nostrils. Chem Senses 17:233-244.

Laing DG (1983) Natural sniffing gives optimum odour perception for humans. Perception 12:99-117.

Mainland J, Sobel N (2006) The sniff is part of the olfactory percept. Chem Senses 31:181-196.

Mainland JD, Johnson BN, Khan R, Ivry RB, Sobel N (2005) Olfactory impairments in patients with unilateral cerebellar lesions are selective to inputs from the contralesional nostril. J Neurosci 25:6362-6371.

Marcell MM, Borella D, Greene M, Kerr E, Rogers S (2000) Confrontation naming of environmental sounds. J Clin Exp Neuropsychol 22:830-864.

Marchetti C, Carey D, Della Sala S (2005) Crossed right hemisphere syndrome following left thalamic stroke. J Neurol 252:403-411.

Mateer C (1978) Asymmetric effects of thalamic stimulation on rate of speech. Neuropsychologia 16:497-499.

McBride SA, Slotnick B (1997) The olfactory thalamocortical system and odor reversal learning examined using an asymmetrical lesion paradigm in rats. Behav Neurosci 111:1273-1284.

Murakami M, Kashiwadani H, Kirino Y, Mori K (2005) State-dependent sensory gating in olfactory cortex. Neuron 46:285-296.

Ojemann GA (1977) Asymmetric function of the thalamus in man. Ann N Y Acad Sci 299:380-396.

Ojemann GA, Van Buren JM (1967) Respiratory, heart rate, and GSR responses from human diencephalon. Arch Neurol 16:74-88.

Plailly J, Howard JD, Gitelman DR, Gottfried JA (2008) Attention to odor modulates thalamocortical connectivity in the human brain. J Neurosci 28:5257-5267.

Porter J, Anand T, Johnson B, Khan RM, Sobel N (2005) Brain mechanisms for extracting spatial information from smell. Neuron 47:581-592.

Potter H, Butters N (1980) An assessment of olfactory deficits in patients with damage to prefrontal cortex. Neuropsychologia 18:621-628.

Prencipe M, Ferretti C, Casini AR, Santini M, Giubilei F, Culasso F (1997) Stroke, disability, and dementia: results of a population survey. Stroke 28:531-536.

Price JL (1985) Beyond the primary olfactory cortex: olfactory-related areas in the neocortex, thalamus and hypothalamus. Chem Senses 10:239-258.

Price JL, Slotnick BM (1983) Dual olfactory representation in the rat thalamus: an anatomical and electrophysiological study. J Comp Neurol 215:63-77.

Principato JJ, Ozenberger JM (1970) Cyclical changes in nasal resistance. Arch Otolaryngol 91:71-77.

Ross SM (2003) Peirce's criterion for the elimination of suspect experimental data. J Eng Technol 20:38-41.

Rousseaux M, Muller P, Gahide I, Mottin Y, Romon M (1996) Disorders of smell, taste, and food intake in a patient with a dorsomedial thalamic infarct. Stroke 27:2328-2330.

Savic I, Gulyas B (2000) PET shows that odors are processed both ipsilaterally and contralaterally to the stimulated nostril. Neuroreport 11:2861-2866.

Savic I, Gulyas B, Larsson M, Roland P (2000) Olfactory functions are mediated by parallel and hierarchical processing. Neuron 26:735-745.

Schmahmann JD (2003) Vascular syndromes of the thalamus. Stroke 34:2264-2278.

Schoenfeld TA, Cleland TA (2006) Anatomical contributions to odorant sampling and representation in rodents: zoning in on sniffing behavior. Chem Senses 31:131-144.

Schuurman PR, Bruins J, Merkus MP, Bosch DA, Speelman JD (2002) A comparison of neuropsychological effects of thalamotomy and thalamic stimulation. Neurology 59:1232-1239. 
Scott JW, McBride RL, Schneider SP (1980) The organization of projections from the olfactory bulb to the piriform cortex and olfactory tubercle in the rat. J Comp Neurol 194:519-534.

Shipley MT, Ennis M (1996) Functional organization of olfactory system. J Neurobiol 30:123-176.

Slotnick BM, Kaneko N (1981) Role of mediodorsal thalamic nucleus in olfactory discrimination learning in rats. Science 214:91-92.

Slotnick BM, Risser JM (1990) Odor memory and odor learning in rats with lesions of the lateral olfactory tract and mediodorsal thalamic nucleus. Brain Res 529:23-29.

Sobel N, Prabhakaran V, Desmond JE, Glover GH, Goode RL, Sullivan EV, Gabrieli JD (1998) Sniffing and smelling: separate subsystems in the human olfactory cortex. Nature 392:282-286.

Sobel N, Khan RM, Saltman A, Sullivan EV, Gabrieli JD (1999) The world smells different to each nostril. Nature 402:35.

Sobel N, Thomason ME, Stappen I, Tanner CM, Tetrud JW, Bower JM, Sullivan EV, Gabrieli JD (2001) An impairment in sniffing contributes to the olfactory impairment in Parkinson's disease. Proc Natl Acad Sci U S A 98:4154-4159.

Staubli U, Schottler F, Nejat-Bina D (1987) Role of dorsomedial thalamic nucleus and piriform cortex in processing olfactory information. Behav Brain Res 25:117-129.

Sudlow CL, Warlow CP (1997) Comparable studies of the incidence of stroke and its pathological types: results from an international collaboration. Stroke 28:491-499.

Talairach J, Tournoux P (1988) Co-planar stereotaxic atlas of the human brain, Ed 3. Dimensional proportional system: an approach to cerebral imaging. Stuttgart, Germany: Thieme.

Tourbier IA, Doty RL (2007) Sniff magnitude test: relationship to odor identification, detection, and memory tests in a clinic population. Chem Senses 32:515-523.

Uva L, de Curtis M (2005) Polysynaptic olfactory pathway to the ipsi- and contralateral entorhinal cortex mediated via the hippocampus. Neuroscience 130:249-258.

Walker JC, Kendal-Reed M, Hall SB, Morgan WT, Polyakov VV, Lutz RW (2001) Human responses to propionic acid. II. Quantification of breathing responses and their relationship to perception. Chem Senses 26:351-358.

Whelan BM, Murdoch BE, Theodoros DG (2002) A role for the dominant thalamus in language? A linguistic comparison of two cases subsequent to unilateral thalamotomy procedures in the dominant and non-dominant hemispheres. Aphasiology 16:1213-1226.

Wilson B, Cockburn J, Baddeley A, Hiorns R (1989) The development and validation of a test battery for detecting and monitoring everyday memory problems. J Clin Exp Neuropsychol 11:855-870.

Wilson DA (1997) Binaral interactions in the rat piriform cortex. J Neurophysiol 78:160-169.

Zainos A, DeAnda R, Chavez L, Garcia-Munoz M (1984) Turning behavior, barrel rolling, and sensory neglect induced by picrotoxin in the thalamus. Exp Neurol 83:534-547.

Zald DH, Pardo JV (1997) Emotion, olfaction, and the human amygdala: amygdala activation during aversive olfactory stimulation. Proc Natl Acad Sci U S A 94:4119-4124. 